Short title: Brachial cuff reservoir pressure parameters

\title{
Association of brachial-cuff excess pressure with carotid intima-media thickness in Australian adults: a cross-sectional study
}

\author{
Xiaoqing PENG ${ }^{\mathrm{a}, \mathrm{b}}$, Melissa WAKE ${ }^{\mathrm{c}, \mathrm{d}, \mathrm{e}}$, Martin G. SCHULTZ ${ }^{\mathrm{b}}$, David P. BURGNER \\ d,e,f,g, , Petr OTAHAL ${ }^{\mathrm{b}}$, Jonathan P. MYNARD ${ }^{\mathrm{d}, \mathrm{e}, \mathrm{f}}$, Susan ELLUL ${ }^{\mathrm{e}}$, Michael CHEUNG \\ c,d,e, Richard S. LIU ${ }^{\mathrm{d}, \mathrm{e}}$, Markus JUONALA ${ }^{\mathrm{e}, \mathrm{h}, \mathrm{i}}$, James E. SHARMAN ${ }^{\mathrm{b}}$
}

${ }^{a}$ Anhui Medical University, Hefei, China. ${ }^{b}$ Menzies Institute for Medical Research, University of Tasmania, Hobart, Australia. ' University of Auckland, Grafton, Auckland, New Zealand. ${ }^{\mathrm{d} D e p a r t m e n t}$ of Paediatrics, University of Melbourne, Parkville, Victoria, Australia. 'Murdoch Children's Research Institute, Parkville, Victoria, Australia. ${ }^{\mathrm{f}}$ Royal Children's Hospital, Melbourne, Victoria, Australia. 'Department of Paediatrics, Monash University, Clayton, Victoria, Australia. ${ }^{\mathrm{h}}$ Department of Medicine, University of Turku, Finland. ${ }^{\mathrm{i}}$ Division of Medicine, Turku University Hospital, Turku, Finland.

Author contributions: XP contributed to data collection, analysis and interpretation of data and drafted manuscript; MGS and JES: project conception and study design, interpretation of data and critical revision of manuscript; MW: Health Design Leader Longitudinal Study of Australian Children, principal investigator Child Health CheckPoint, project conception and critical revision of manuscript; $\mathrm{MC}, \mathrm{DB}$ and $\mathrm{MJ}$ : project conception and critical revision of manuscript; JM: suggestion of data analysis and critical revision of manuscript; PO and SE: statistical assistance and critical revision of manuscript; RL: contributed to data collection, advice on data analysis and critical revision of manuscript. 


\section{Sources of Funding}

This work has been supported by the National Health and Medical Research Council of Australia (1041352, 1109355), the Royal Children's Hospital Foundation (2014-241), Murdoch Childrens Research Institute, the University of Melbourne, National Heart Foundation of Australia (100660), Financial Markets Foundation for Children (2014055). The funding bodies played did not play any role in the study. The following authors were supported by the National Health and Medical Research Council of Australia: Early Career Fellowship (1104731) to MGS. Wright Career Development Fellowship (1143510) to JM, Senior Research Fellowships (1046518) to MW and (1064629) to DPB, Postgraduate Research Scholarship (1114567) to RL, Career Development Fellowship (409940) to JS. MW was also supported by Cure Kids, New Zealand, and DB and JM were also supported by Future Leader Fellowship of the National Heart Foundation of Australia (100369 and 101866). MJ was supported by the

Federal Research Grant of Finland to Turku University Hospital, Finnish Cardiovascular Foundation.

\section{Disclosures}

This article uses unit record data from the Longitudinal Study of Australian Children. The study is conducted in partnership between the Department of Social Services (DSS), the Australian Institute of Family Studies (AIFS) and the Australian Bureau of Statistics (ABS). The findings and views reported in this article are those of the author and should not be attributed to DSS, AIFS or the ABS. 
Conflicts of interest

The authors declare no potential conflicts of interest, including no specific financial interests relevant to the subject of this manuscript.

Total word count: 4789 Abstract word count: 246 Figure: 2 Tables: 3

\section{Corresponding author:}

James E. Sharman

Menzies Institute for Medical Research, College of Health and Medicine,

University of Tasmania, Medical Science Building, Liverpool Street, Hobart, 7000,

Australia

Telephone: +61 (0) 362264709

Fax: +61 (0)3 62267704

Email: James.Sharman@utas.edu.au 


\begin{abstract}
Objective. Reservoir pressure parameters (e.g. reservoir pressure [RP] and excess pressure $[\mathrm{XSP}])$ measured using tonometry predict cardiovascular events beyond conventional risk factors. However, the operator-dependency of tonometry impedes widespread use. An operator-independent cuff-based device can reasonably estimate the intra-aortic RP and XSP from brachial volumetric waveforms, but whether these estimates are clinically relevant to preclinical phenotypes of cardiovascular risk has not been investigated.
\end{abstract}

Methods. RP and XSP were derived from brachial volumetric waveforms measured using cuff oscillometry (SphygmoCor XCEL) in 1691 mid-life adults from the CheckPoint study (a population-based cross-sectional study nested in the Longitudinal Study of Australian Children). Carotid intima-media thickness (carotid IMT, n=1447) and carotid-femoral pulse wave velocity (PWV, n=1632) were measured as preclinical phenotypes of cardiovascular risk. Confounders were conventional risk factors that were correlated with both exposures and outcomes or considered as physiologically important.

Results. There was a modest association between XSP and carotid IMT $(\beta=3.69 \mu \mathrm{m}$, 95\%CI, 1.06 to 6.32 , partial $\mathrm{R}^{2}=0.7 \%$ ) after adjusting for age, sex, body mass index, heart rate, smoking, diabetes, high-density lipoprotein cholesterol and mean arterial pressure. Neither RP nor XSP were associated with PWV in the similarly adjusted models $\left(\beta=-0.47 \mathrm{~cm} / \mathrm{s}, 95 \% \mathrm{CI},-1.15\right.$ to 0.20 , partial $\mathrm{R}^{2}=0.2 \%$ for $\mathrm{RP}$, and $\beta=0.04 \mathrm{~cm} / \mathrm{s}$, 95\%CI, -0.59 to 0.67 , partial $\mathrm{R}^{2}=0.01 \%$ for $\left.\mathrm{XSP}\right)$. 
Conclusion. Cuff-based XSP associates with carotid IMT independent of conventional risk factors, including traditional $\mathrm{BP}$, but the association was weak, indicating that further investigation is warranted to understand the clinical significance of reservoir pressure parameters.

Keywords. Reservoir, blood pressure monitor, atherosclerosis, cardiovascular risk. 


\section{Introduction}

Cardiovascular disease remains the largest cause of mortality worldwide, and high blood pressure (BP) is a leading risk factor. ${ }^{1-3}$ Traditional BP is derived from the estimation of the peak (systolic BP), nadir (diastolic BP) and area (MAP, mean arterial pressure) of brachial arterial BP waveforms. Several theoretical constructs have been suggested to explain the physiology underlying the BP waveform. ${ }^{4}$ One such explanation is the reservoir-excess pressure model, which proposes that the total BP waveform comprises a reservoir pressure (RP, determined by the systemic arterial compliance and peripheral resistance) and an excess pressure (XSP, related to the local wave propagation) component. ${ }^{5}$ These variables are associated with arterial function and structure, ${ }^{6,7}$ and have been shown to predict cardiovascular morbidity and mortality beyond conventional risk factors (including the systolic rate constant), ${ }^{7-13}$ thus, may be risk factors for vascular injury. In these studies the aforementioned reservoir pressure parameters were measured using tonometry at the carotid or radial arteries, but this technique is operator-dependent ${ }^{14}$ and has not yet been adopted in clinical settings.

Oscillometric cuff devices are routinely used for BP assessment and this may offer a user-friendly and operator-independent method to undertake more widespread measurement of reservoir pressure parameters. We recently compared reservoir pressure parameters derived non-invasively using a cuff device (from brachial volumetric waveforms) with aortic reservoir pressure parameters recorded invasively by catheter. This study found acceptable concordance of the cuff-based measures with intra-aortic measures of RP and XSP (mean differences were $-8 \pm 4 \mathrm{mmHg} / \mathrm{s}$ and $1 \pm 2$ 
$\mathrm{mmHg} / \mathrm{s}$, and intra-class correlation coefficients were $0.66,95 \%$ confidence interval [CI] 0.57 to 0.73 and $0.60,95 \%$ CI 0.49 to 0.68$).{ }^{15}$ These prior findings imply that the cuff-based method may be useful for deriving reservoir pressure parameters in clinical settings. However, the clinical utility of brachial-cuff reservoir pressure parameters remains to be determined. Therefore, the aim of this study was to examine the independent associations between brachial-cuff reservoir pressure parameters and preclinical phenotypes of cardiovascular risk, including pre-atherosclerosis and aortic stiffness, in a large cohort of Australian adults.

\section{Methods}

Study design and population. Participants were the adult guardians (usually mothers) who accompanied child participants in the Child Health CheckPoint study (CheckPoint). CheckPoint was a cross-sectional comprehensive assessment of physical health and biomarkers within the Longitudinal Study of Australian Children (LSAC) birth cohort, conducted between LSAC's sixth and seventh waves. LSAC applied a two-stage sampling design. The first phase was random selection of ten percent of all Australian postcodes (stratified by state and urban/rural domicile). The second phase involved selection of children from the Medicare database. 8928 healthy infants at age $0-1$ years in 2014 and their families were recruited for LSAC birth cohort. The response rate was $57.2 \%$ for wave 1 in 2004 , of whom $73.7 \%$ were retained for wave 6 in $2014 .{ }^{16}$ CheckPoint families were from wave 6 of LSAC. 1874 children and one of the adult guardians attended for assessment. Details of the study population and health assessment protocols in CheckPoint study have been published. ${ }^{17,18}$ The study protocol 
was in accordance to the ethical guidelines of the 1975 Declaration of Helsinki and approved by the Royal Children's Hospital Melbourne Human Research Ethics Committee (33225D) and Australian Institute of Family Studies Ethics Committee. Participants gave written informed consent.

Study procedures. The CheckPoint data collection spanned from February 2015 to March 2016. Participants attended one of 15 assessment centres nationwide $(n=1509$, $80 \%$ ) or, if unable to attend, a shorter home visit was undertaken at the participant's home $(n=365,20 \%)$. Measurements of carotid intima-media thickness (carotid IMT) or lipids (i.e. total cholesterol, high-density lipoprotein cholesterol, low-density lipoprotein cholesterol and triglycerides) were only obtained on participants who attended an assessment centre because these measurements were not feasible at home visits. ${ }^{19}$ All the other measures were obtained in both assessment centre and home visit settings.

Reservoir pressure parameters. Brachial BP and brachial volumetric waveforms were acquired in triplicate using an oscillometric cuff device (SphygmoCor XCEL, AtCor Medical Pty Ltd., West Ryde, Australia) in the supine position after seven minutes rest. Eight participants did not complete the measurement of brachial BP and brachial volumetric waveforms. Brachial volumetric waveforms were ensemble averaged by the built-in software before calibration and consequent derivation of reservoir pressure parameters. A quality check of brachial BP waveforms was performed based on average pulse height ( $>80$ units), pulse height variation $(\leq 5 \%)$, 
diastolic variation $(\leq 5 \%)$, shape deviation $(\leq 4 \%)$, operator index (default evaluated and reported by SphymoCor XCEL, $\geq 75$ ) and systolic BP between 50 and $200 \mathrm{mmHg}$. Only $\mathrm{BP}$ waveforms that passed all the quality criteria were eligible for inclusion and data from 175 participants were excluded. For each of the remaining 1691 participants, the first eligible brachial BP waveform was calibrated with the average of three brachial systolic and diastolic BPs. Reservoir pressure parameters were calculated using a customised MATLAB program (Mathworks, Inc, Natick, MA) with the pressure-only approach, by solving equation (1). ${ }^{7}$

$$
\frac{d P_{\text {reservoir }}}{d t}=S c\left(P-P_{\text {reservoir }}\right)-D c\left(P_{\text {reservoir }}-P_{\infty}\right)
$$

where $P$ is measured total pressure, $P_{\text {reservoir }}$ is RP, and $P_{\infty}$ is the arterial asymptotic pressure. $S c$ and $D c$ are the systolic and diastolic rate constants, relating to the speed of the upstroke and downstroke on the BP waveform respectively. ${ }^{5} \mathrm{XSP}$ is defined as the difference between the measured total pressure and RP. Figure 1 is an example BP waveform that shows the RP and XSP components. RP and XSP magnitudes are quantified via both the peak and integral of the respective waveforms. The RP algorithm expects waveforms to have an exponential pressure decay during diastole and therefore can generate non-physiological values of $\mathrm{P}_{\infty}$ (i.e. that is greater than diastolic $\mathrm{BP}$ ) in cases with an additional small upslope after the nadir of the BP waveform in diastole. This problem appears to arise because the algorithm was applied to the ensemble averaged waveform without consideration of cardiac duration. Waveform modification was performed to resolve the problem, firstly by removal of the small upslope occurring 
at end diastole and then re-applying the algorithm to derive reservoir pressure parameters.

Preclinical phenotypes of cardiovascular risk. Carotid pre-atherosclerosis was determined by common carotid artery IMT using a high-performance $10 \mathrm{MHz}$ L-RS vascular ultrasound probe (Vivid I Bt06, GE) in accordance with recommendations of the American Society of Echocardiography and Mannheim Consensus statements. ${ }^{20}$ Images of the right common carotid artery were captured over 5-10 cardiac cycles (tracked using three-lead ECG) at $10 \mathrm{~mm}$ proximal to the carotid bulb in supine position. Ultrasonography was performed by six trained researchers. The inter- and intraoperator reliability of measurements was tested in 105 images. The within-observer coefficient of variation was $6.5 \%$ for mean carotid IMT values, and the betweenobserver coefficient of variation was $9.5 \%$. Within-observer intra-class correlations were 0.71 (95\% CI, 0.63-0.78), and between-observer intra-class correlations were 0.64 (95\% CI, 0.54-0.74). Inter- and intra-operator reliability of the carotid IMT measurement were comparable to other published results ${ }^{21}$. B-mode ultrasound cine loops were captured in triplicate. The images were analysed using Carotid Analyzer (Medical Imaging Applications, Coralville, IA, USA) for semi-automated border detection, and this was blinded to reservoir pressure parameters value. The carotid IMT was measured as the mean thickness in micrometers of 3 to 5 frames of the entire carotid IMT measurement over the 5- $10 \mathrm{~mm}$ section. The average of three carotid IMT measurements was used in the analysis. 
Aortic stiffness was measured by carotid-femoral pulse wave velocity (PWV) in triplicate according to the consensus guidelines ${ }^{22}$ using SphygmoCor XCEL. A cuff was placed around the participant's upper thigh to capture the femoral artery pulse and a tonometer (Millar Micro-tip SPT-transducer, Houston, USA) was used to simultaneously record the carotid artery pulse. PWV was calculated as the distance between carotid and femoral recording sites divided by the pulse transit time. The distance was defined as the distance from sternal notch to top edge of femoral cuff minus the distance from carotid artery to sternal notch. Details of carotid IMT and PWV measurements have been published. ${ }^{23}$

Other sample characteristics. Anthropometry was performed with the participants in light clothing and without shoes. Height was measured in duplicate using a portable stadiometer. If the difference between the two measurements was greater than $0.5 \mathrm{~cm}$, a third measurement was taken and the mean of all measurements used. Weight was measured using an InBody230 bio-electrical impedance analysis scale (Biospace Co. Ltd. Seoul, South Korea). Body mass index (BMI) was calculated as weight $(\mathrm{kg}) /$ height ${ }^{2}\left(\mathrm{~m}^{2}\right)$, and overweight was defined as BMI greater than $25 \mathrm{~kg} / \mathrm{m}^{2}{ }^{24}$ The disadvantage index was a standardised score by geographic area compiled from census data to numerically summarize the social and economic conditions of Australian neighborhoods ${ }^{25}$, and extracted from the self-reported questionnaire collected in CheckPoint. Lipids including total cholesterol, high-density lipoprotein cholesterol, low-density lipoprotein cholesterol and triglycerides were measured in venous blood after at least two hours fasting via the Nightingale ${ }^{\circledR}$ Nuclear Magnetic Resonance 
metabolomics platform according to the 2017-version quantification algorithm. ${ }^{26}$ Information on smoking status was extracted from the self-reported questionnaire collected in LSAC wave 6, one year earlier. Smoking was defined based as consuming $\geq 1$ cigarette per day. Diabetes status was extracted from the self-reported questionnaire collected in CheckPoint and defined as either type 1 or type 2 diabetes. Heart rate was obtained during BP measurement using SphygmoCor XCEL. MAP was calculated as diastolic $\mathrm{BP}+1 / 3$ pulse pressure (PP). Hypertension was defined according to $\mathrm{BP}$ $\geq 140 / 90 \mathrm{mmHg}$ from the averaged triplicate BPs.

Statistical analysis. Continuous data were presented as mean (SD) and categorical data as \%. 'Exposures' were RP peak, RP integral, XSP peak and XSP integral. 'Outcomes' were carotid IMT and PWV. Uni- and multi-variable regression analyses were performed to examine the associations between exposures and outcomes. Conventional risk factors ${ }^{27}$ that were correlated with both exposures and outcomes $(r>0.1)$ or considered as physiologically important (i.e. heart rate), were included as confounders in the adjusted models. Altogether, sex, age, BMI, heart rate, smoking, diabetes and high-density lipoprotein cholesterol were included a priori in the basic-adjusted models. Each of systolic BP, diastolic BP and MAP were additionally added in the fullyadjusted models to investigate the role of traditional BP on the associations between outcomes and exposures. Partial coefficients of determination (partial $\mathrm{R}^{2}$ ) are presented as the percentage variance in outcomes explained by each risk factor. No evidence of interaction between exposures and sex in explaining outcomes was found. Thus, 
females and males were combined in analyses. Data were analysed using Stata 1.5 (StataCorp LP, TX, USA).

\section{Results}

Characteristics of the study population. Figure 2 shows the participant flow and Table 1 presents the participant characteristics. Participants were between 28 and 71 years of age and predominantly female. Prevalence of hypertension, smoking and diabetes were low, but the majority of participants $(n=1049,62 \%)$ were overweight. The disadvantage index was higher than a national mean of 1000 and SD of 100, indicating that participants was on average less disadvantaged than the general Australian population. Conventional risk factors including age, sex, traditional BP, BMI, diabetes and high-density lipoprotein cholesterol were correlated in the expected directions with both carotid IMT and PWV, but smoking was not significantly correlated with carotid IMT $(\beta=1.12 \mu \mathrm{m}, 95 \% \mathrm{CI},-12.00$ to $14.23, \mathrm{p}=0.867)$ and the relationship with PWV was borderline ( $\beta=-8.8 \mathrm{~cm} / \mathrm{s}, 95 \% \mathrm{CI},-26.7$ to $9.2, \mathrm{p}=0.088)$.

\section{Associations between reservoir pressure parameters and preclinical phenotypes of}

cardiovascular risk. The effect of systolic $\mathrm{BP}$, diastolic $\mathrm{BP}$ or MAP on the associations between reservoir pressure parameters and preclinical phenotypes of cardiovascular risk were similar for each $\mathrm{BP}$ variable. Therefore, results were only presented for MAP as MAP is the key determinant of large artery stiffness and the BP variable recommended by expert consensus to be considered as a potential confounder in statistical analyses. $^{28}$ 
Carotid pre-atherosclerosis. Table 2 summarises the associations between reservoir pressure parameters and carotid IMT in the uni- and multi-variable regression models. RP peak, RP integral, XSP peak and XSP integral were positively associated with carotid IMT in the univariable regression models. XSP peak and XSP integral remained to be positively associated with carotid IMT independent of age, sex, BMI, heart rate, smoking, diabetes, high-density lipoprotein cholesterol and MAP in the fully-adjusted model. However, the relationship was weak, only explaining less than $1 \%$ of the variance in carotid IMT. RP peak and RP integral did not contribute additional meaningful variance in carotid IMT in the fully-adjusted models.

Aortic stiffness. Table 3 summarises the associations between reservoir pressure parameters and PWV in the uni- and multi-variable regression models. RP peak, RP integral, XSP peak and XSP integral were positively associated with PWV in univariable models. After adjusting for age, sex, BMI, heart rate, smoking, diabetes and high-density lipoprotein in the basic-adjusted model, the positive association between RP and PWV persisted. However, the association substantially attenuated after further adjusting for MAP in fully-adjusted model. XSP peak and XSP integral did not contribute additional meaningful variance in PWV in the adjusted models.

\section{Discussion}

This study is the first to apply a clinically convenient cuff approach to measure RP and XSP and investigate the associations with preclinical phenotypes of cardiovascular risk (carotid IMT and PWV) in a large adult population. The novel findings were that XSP 
was associated with carotid IMT after adjustment for confounders, and further, that the association between RP and PWV was substantially influenced by traditional BP. These findings demonstrate in principle that brachial-cuff XSP provides useful information on cardiovascular risk above and beyond conventional risk factors among adults.

The findings should be interpreted in light of the relatively weak associations between reservoir pressure parameters and the preclinical cardiovascular phenotypes, which may be due to the lack of precision in deriving reservoir pressure parameters using the cuff-based technique employed in this study. This approach involved recording the brachial artery volumetric waveform at sub-diastolic (low) pressure and then applying algorithms to derive reservoir pressure parameters. Unfortunately, waveform features are dampened when recorded at low pressure, which means that some reservoir pressure parameters cannot be accurately reproduced (e.g. the systolic and diastolic rate constants), and this leads to higher variance in derivation of XSP and RP. ${ }^{15}$ Altogether, this indicates that refinement of the cuff-based method to derive higher precision for measurement of reservoir pressure parameters is probably needed before testing for potential clinical utility.

Notwithstanding, the observed association between brachial-cuff XSP and carotid IMT was similar to that reported in the CAFÉ study, which measured XSP integral at the radial artery using tonometry. ${ }^{7}$ Although XSP is generally lower at the brachial artery than the radial artery, ${ }^{29}$ the concordant findings suggest that the similar prognostic value of XSP for predicting carotid IMT may be achievable at either measurement site. ${ }^{7,9}$ Our 
new observation was that even though XSP marginally contributed to the total explainable variance in carotid IMT, this was independent of conventional risk factors including traditional BP. However, the underlying physiological reasons explaining the association between reservoir pressure parameters and carotid IMT is unclear and requires further investigation.

In keeping with the findings of a previous catheterisation laboratory study, ${ }^{6}$ we found a positive association between RP and PWV, but this association no longer existed after adjusting for traditional BP. The association between RP and PWV in the basic-adjusted models are plausible because there are overlapping arterial properties represented by both RP and PWV. RP is a systemic measure that is dependent on multiple factors, including left ventricular stroke volume, aortic diameter and stiffness, systemic arterial compliance and peripheral resistance, ${ }^{5}$ and is relatively constant between central and peripheral large arteries. ${ }^{29}$ On the other hand, PWV is a regional measure of arterial stiffness over a defined (central) arterial region. ${ }^{22}$ The association between RP and PWV was slightly attenuated after adjusting for age, sex, BMI, heart rate, smoking, diabetes and high-density lipoprotein cholesterol in the basic-adjusted models, and this is probably because conventional risk factors are associated with arterial function and structure, which are the determinants of both RP and PWV. ${ }^{28,30}$ Furthermore, the association was substantially attenuated after inclusion of MAP in the fully-adjusted models, which may be driven by two factors. Firstly, a large proportion of the contribution to MAP (greater than $70 \%$ ) is attributable to RP integral, ${ }^{29}$ and this will lead to a strong correlation between MAP and RP. Secondly, distending pressure is a 
major determinant of PWV, and thus MAP strongly associates with PWV. ${ }^{31,32}$ The role of traditional $\mathrm{BP}$ on the associations between RP and PWV might be mediating or confounding, however, this study cannot confirm these causal pathways.

The strengths of our study include a large nationally-derived population sample with a wide range of age and high-quality measures. The study also has wide social and geographic representation across Australia, but the sample is under-represented by low social-economic position families ${ }^{18}$. The study also has wide social and geographic representation across Australia, but the sample is under-represented by low socialeconomic position families ${ }^{18}$. Smoking was not associated with carotid IMT, which was unexpected and could suggest a lack of generalizability. However, we speculate that this may be because smoking status was self-reported in LSAC wave 6, which was one year earlier than the other data collected. If there were changes in smoking status by the time carotid IMT was measured, this could have influenced this unexpected finding, particularly since the cardiovascular effects of smoking exposure can be detected within a relatively short period (e.g. within 6 months ${ }^{33}$ ). Another potential limitation is that reservoir pressure parameters were measured in the supine position, whereas, clinical BP is usually measured whilst seated. Furthermore, calculation of reservoir pressure parameters relied on a pressure-only approach (no flow), which is a substitute of the standard pressure-flow method and involves additional assumptions. ${ }^{34}$ Nevertheless, this method has been shown to produce substantially equivalent reservoir pressure parameters to the pressure-flow method ${ }^{34}$ calculated reservoir pressure parameters. ${ }^{34}$ 
In conclusion, we found that brachial-cuff reservoir pressure parameters were independently associated with a preclinical phenotype of cardiovascular risk separate from conventional cardiovascular risk factors among middle-age adults. The magnitude of the association between brachial-cuff XSP and carotid IMT was weak and clinical relevance remains unclear. Furthermore, brachial-cuff RP was related to PWV, but this association was substantially influenced by conventional BP. These findings suggest that a clinically convenient cuff approach to measuring reservoir pressure parameters may have potential clinical utility for better cardiovascular risk assessment, however, further prospective clinical studies are required.

\section{Acknowledgements}

We are grateful to Mr Xun Yang, HeFei University of Technology, China for providing some technical assistance.

\section{References}

1. Lewington S, Clarke R, Qizilbash N, et al. Age-specific relevance of usual blood pressure to vascular mortality: a meta-analysis of individual data for one million adults in 61 prospective studies. Lancet. 2002;360(9349):1903-13.

2. Kannel WB. Role of blood pressure in cardiovascular disease: The Framingham Study. Angiology. 1975;26(1):1-14.

3. Collaborators GBDRF. Global, regional, and national comparative risk assessment of 79 behavioural, environmental and occupational, and metabolic risks or 
clusters of risks, 1990-2015: a systematic analysis for the Global Burden of Disease Study 2015. Lancet. 2016;388(10053):1659-1724.

4. Segers P, O'Rourke MF, Parker K, et al. Towards a consensus on the understanding and analysis of the pulse waveform: Results from the 2016 Workshop on Arterial Hemodynamics: Past, present and future. Artery Res. 2017;18:75-80.

5. Wang JJ, O'Brien AB, Shrive NG, et al. Time-domain representation of ventricular-arterial coupling as a windkessel and wave system. Am J Physiol Heart Circ Physiol. 2003;284(4):H1358-68.

6. Davies JE, Baksi J, Francis DP, et al. The arterial reservoir pressure increases with aging and is the major determinant of the aortic augmentation index. American Journal of Physiology-Heart and Circulatory Physiology. 2010;298(2):H580-H586.

7. Davies JE, Lacy $\mathrm{P}$, Tillin $\mathrm{T}$, et al. Excess pressure integral predicts cardiovascular events independent of other risk factors in the conduit artery functional evaluation substudy of Anglo-Scandinavian Cardiac Outcomes Trial. Hypertens. 2014;64(1):60-8.

8. Wang WT, Sung SH, Wang JJ, et al. Excess Pressure Integral Predicts LongTerm All-Cause Mortality in Stable Heart Failure Patients. Am J Hypertens. 2017;30(3):271-278.

9. Cheng HM, Chuang SY, Wang JJ, et al. Prognostic significance of mechanical biomarkers derived from pulse wave analysis for predicting long-term cardiovascular mortality in two population-based cohorts. Int J Cardiol. 2016;215:388-95. 
10. Hametner B, Wassertheurer S, Hughes AD, et al. Reservoir and excess pressures predict cardiovascular events in high-risk patients. Int J Cardiol. 2014;171(1):31-6.

11. Parragh S, Hametner B, Mayer C, et al. U-shaped relationship of reservoir pressure to cardiovascular events in patients with heart failure with reduced ejection fraction. Artery Res. 2016;16:55.

12. Narayan O, Davies JE, Hughes AD, et al. Central aortic reservoir-wave analysis improves prediction of cardiovascular events in elderly hypertensives. Hypertens. 2015;65(3):629-35.

13. Schneider A, Krauze T, Minczykowski A, et al. Arterial excessreservoir pressure integral as a predictor of cardiovascular complications in patients with acute coronary syndrome. Pol Arch Intern Med. 2018;128(4):228-234.

14. McEniery CM, Cockcroft JR, Roman MJ, et al. Central blood pressure: current evidence and clinical importance. Eur Heart J. 2014;35(26):1719-25.

15. Peng X, Schultz MG, Picone DS, et al. Non-invasive measurement of reservoir pressure parameters from brachial-cuff blood pressure waveforms. J Clin Hypertens (Greenwich). 2018;20(12):1703-1711.

16. Norton A and Monahan K. Wave 6 weighting and non-response. LSAC technical paper. 2015;15.

17. Wake M, Clifford S, York E, et al. Introducing Growing Up in Australia's Child Health CheckPoint.

18. Services DoS. Longitudinal Study of Australian Children Release 7.0 (Waves 1-7), Additional Files. 2018. 
19. Liu R, Dunn S, Grobler A, et al. Carotid artery intima-media thickness, distensibility, and elasticity: population epidemiology and concordance in Australian 11-12 year old Australians and their parents. BMJ Open. 2019:e020264.

20. Stein JH, Korcarz CE, Hurst RT, et al. Use of carotid ultrasound to identify subclinical vascular disease and evaluate cardiovascular disease risk: a consensus statement from the American Society of Echocardiography Carotid Intima-Media Thickness Task Force endorsed by the Society for Vascular Medicine. Journal of the American Society of Echocardiography. 2008;21(2):93-111.

21. Doyon A, Kracht D, Bayazit AK, et al. Carotid artery intima-media thickness and distensibility in children and adolescents: reference values and role of body dimensions. Hypertens. 2013;62(3):550-6.

22. Van Bortel LM, Laurent S, Boutouyrie P, et al. Expert consensus document on the measurement of aortic stiffness in daily practice using carotid-femoral pulse wave velocity. J Hypertens. 2012;30(3):445-448.

23. Clifford S DS, Wake M. Growing Up in Australia's Child Health CheckPoint cohort summary and methodology. BMJ Open. 2018;in press.

24. Organization WH. global database on body mass index; 2010. Global Database on Body Mass Index. 2016.

25. Statistics. ABo. Census of population and housing: socie-economics indexes for area (SEIFA). 2011Cat. No. 2033.0.55.001. 2011. 
26. Würtz P, Kangas AJ, Soininen P, et al. Quantitative serum nuclear magnetic resonance metabolomics in large-scale epidemiology: a primer on-omic technologies. American journal of epidemiology. 2017;186(9):1084-1096.

27. Yusuf S, Reddy S, Ônpuu S, et al. Global burden of cardiovascular diseases: part I: general considerations, the epidemiologic transition, risk factors, and impact of urbanization. Circulation. 2001;104(22):2746-2753.

28. Townsend RR, Wilkinson IB, Schiffrin EL, et al. Recommendations for Improving and Standardizing Vascular Research on Arterial Stiffness: A Scientific Statement From the American Heart Association. Hypertens. 2015;66(3):698-722.

29. Peng X, Schultz MG, Picone DS, et al. Arterial reservoir characteristics and central-to-peripheral blood pressure amplification in the human upper limb. $J$ Hypertens. 2017;35(9):1825-1831.

30. Peng X. Non-invasive reservoir pressure parameters: measurement and clinical relevance.Unpublished doctoral thesis. 2019.

31. Ye C, Pan Y, Xu X, et al. Pulse wave velocity in elastic and muscular arteries: tracking stability and association with anthropometric and hemodynamic measurements. Hypertens Res. 2016;39(11):786-791.

32. Collaboration RVfAS. Determinants of pulse wave velocity in healthy people and in the presence of cardiovascular risk factors:'establishing normal and reference values'. European heart journal. 2010;31(19):2338-2350.

33. Rigotti NA and Clair C. Managing tobacco use: the neglected cardiovascular disease risk factor. European heart journal. 2013;34(42):3259-3267. 
34. Aguado-Sierra J, Davies JE, Hadjiloizou N, et al. Reservoir-wave separation and wave intensity analysis applied to carotid arteries: a hybrid 1D model to understand haemodynamics. Conf Proc IEEE Eng Med Biol Soc. 2008;2008:1381-4. 


\section{Figure legends}

Figure 1. Blood pressure waveform ( _ $)$ with example reservoir pressure parameters. The reservoir pressure (.......) and excess pressures $\left(-\right.$. $\left.^{-}\right)$are expressed in both peak and integral, where the peak refers to the highest value and integral refers to the area under curve.

Figure 2. Summary of participant flow. One child and one of adult guardians from the Longitudinal Study of Australian Children (LSAC) families attended CheckPoint, but only data from adult guardians were used in this study. Waveform modification refers to removing the additional small upslope after the nadir of the BP waveform in diastole, and details are in the Methods 
Figure 1

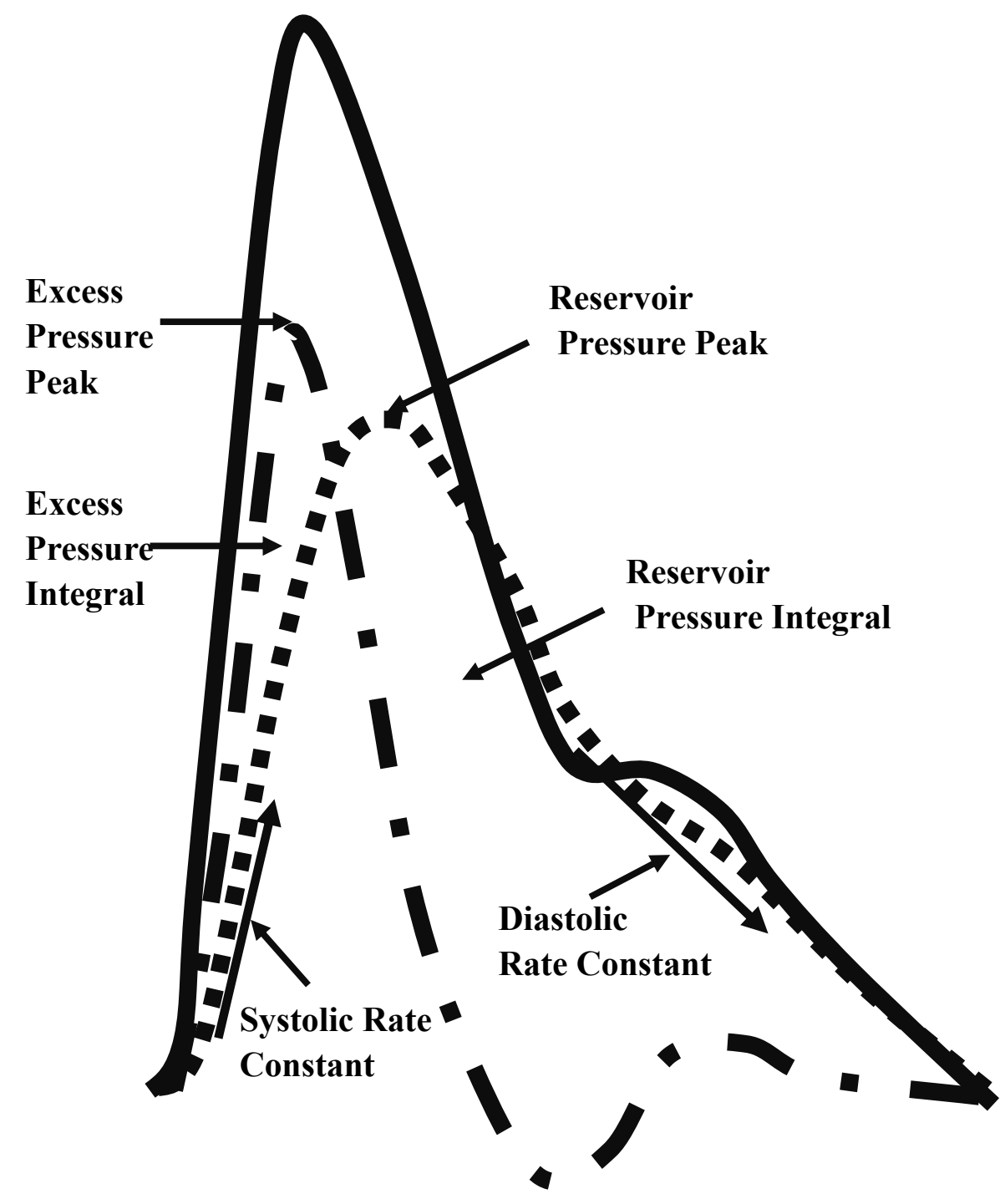

Figure 1. Blood pressure waveform ( _ _ ) with example reservoir pressure parameters. The reservoir pressure (...... ) and excess pressures (- • - ) are expressed in both peak and integral, where the peak refers to the highest value and integral refers to the area under curve. 


\section{Figure 2}

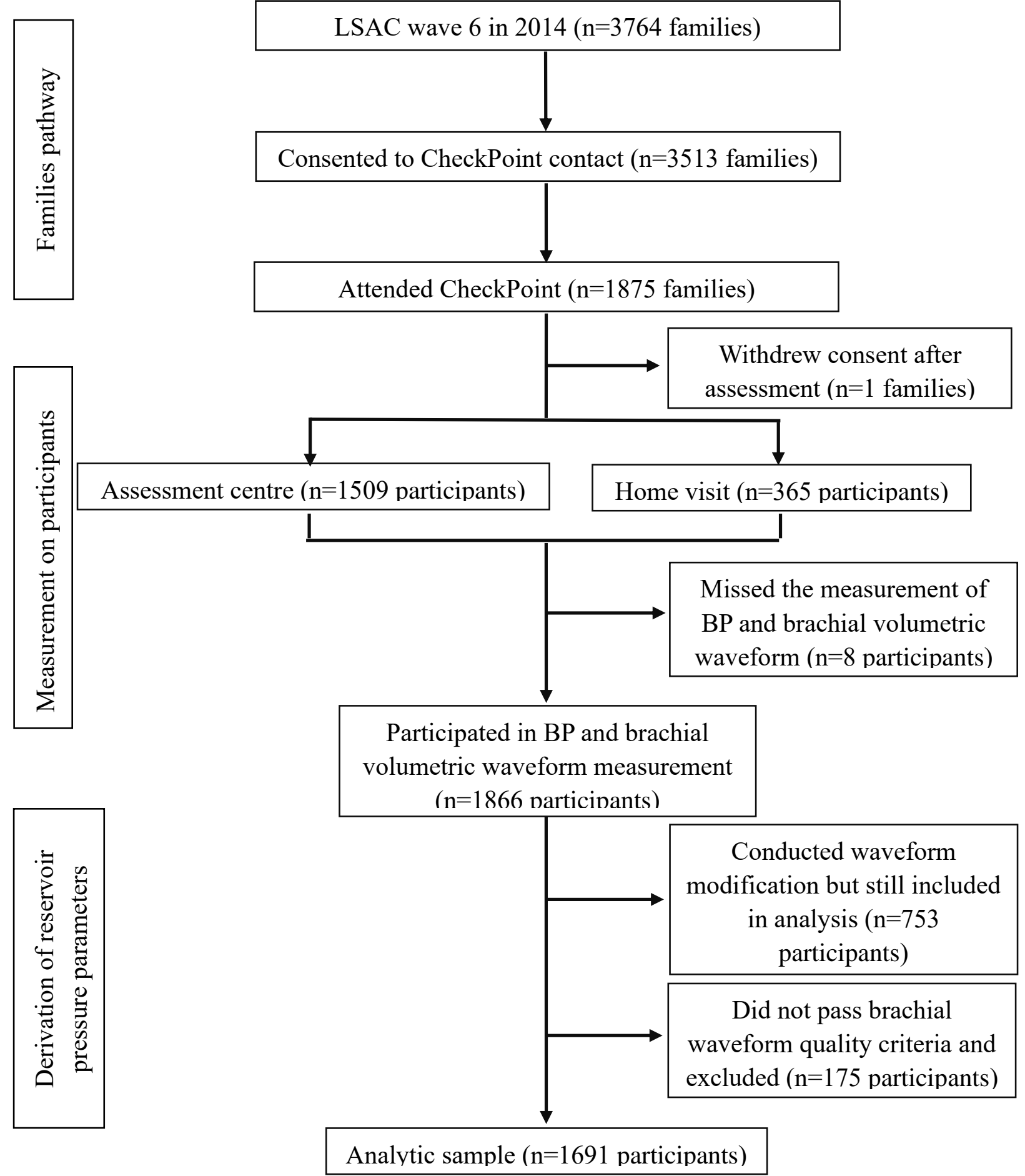


Figure 2. Summary of participant flow. One child and one of adult guardians from the Longitudinal Study of Australian Children (LSAC) families attended CheckPoint, but only data from adult guardians were used in this study. Waveform modification refers to removing the additional small upslope after the nadir of the BP waveform in diastole, and details are in the Methods. 
Table 1. Characteristics of the study participants.

\begin{tabular}{|c|c|}
\hline Variable & Mean (SD) or \% \\
\hline $\mathrm{n}$ & 1691 \\
\hline Age (years) & $44(5)$ \\
\hline Sex $($ men \%) & 11 \\
\hline Disadvantage index & $1024(60)$ \\
\hline Body mass index $\left(\mathrm{kg} / \mathrm{m}^{2}\right)$ & $28(6)$ \\
\hline Brachial systolic blood pressure (mmHg) & $119(13)$ \\
\hline Brachial diastolic blood pressure $(\mathrm{mmHg})$ & $73(9)$ \\
\hline Mean arterial pressure $(\mathrm{mmHg})$ & $87(10)$ \\
\hline Pulse pressure $(\mathrm{mmHg})$ & $46(7)$ \\
\hline Hypertension (yes \%) & 9 \\
\hline Carotid intima-media thickness $(\mu \mathrm{m})$ & $566(75)$ \\
\hline Carotid-femoral pulse wave velocity $(\mathrm{cm} / \mathrm{s})$ & $687(113)$ \\
\hline Heart rate $(\mathrm{bpm})$ & $64(10)$ \\
\hline RP peak (mmHg) & $29(8)$ \\
\hline RP integral (mmHg/s) & $8(2)$ \\
\hline XSP peak (mmHg) & $27(9)$ \\
\hline XSP integral (mmHg/s) & $3(2)$ \\
\hline Smoking (yes \%) & 11 \\
\hline Diabetes (yes \%) & 2 \\
\hline \multicolumn{2}{|l|}{ Lipid profile (mmol/L) } \\
\hline High-density lipoprotein cholesterol & $1.5(0.4)$ \\
\hline Low-density lipoprotein cholesterol & $1.7(0.4)$ \\
\hline Triglycerides & $1.5(0.8)$ \\
\hline Total cholesterol & $4.8(0.9)$ \\
\hline
\end{tabular}

RP, reservoir pressure; XSP, excess pressure. Hypertension was defined based on systolic $\mathrm{BP} \geq 140$ $\mathrm{mmHg}$ and/or diastolic $\mathrm{BP} \geq 90 \mathrm{mmHg}$ of the averaged triplicate values measured at assessment centre. 
Table 2. Uni- and multi-variable regression models on the associations between reservoir pressure parameters and carotid intima-media thickness.

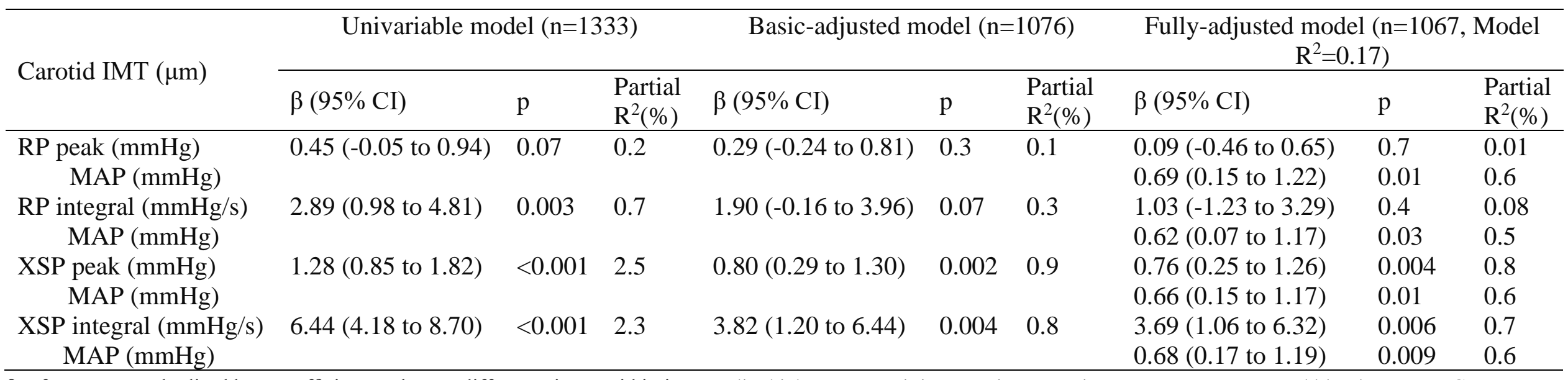

$\beta$ refers to unstandardised beta coefficient as the $\mu \mathrm{m}$ difference in carotid intima-media thickness per unit increase in reservoir pressure parameters and blood pressure. CI,

confidence interval. $p$ value is for the unstandardised $\beta$. Model $R^{2}$ is the unadjusted model $R^{2}$ as a proportion. Partial $R^{2}(\%)$ is the proportion of total variance in carotid intimamedia thickness explained by individual risk factor. Basic-adjusted models adjust for age, sex, BMI, heart rate, smoking, diabetes and high-density lipoprotein cholesterol. Fullyadjusted models have an additional mean arterial pressure or pulse pressure above the basic-adjusted models. RP, reservoir pressure; XSP, excess pressure; and, MAP, mean arterial pressure. 
Table 3. Uni- and multi-variable regression models on the associations between reservoir pressure parameters and carotid-femoral pulse wave velocity.

\begin{tabular}{|c|c|c|c|c|c|c|c|c|c|}
\hline \multirow{2}{*}{$\mathrm{PWV}(\mathrm{cm} / \mathrm{s})$} & \multicolumn{3}{|c|}{ Univariable model $(\mathrm{n}=1546)$} & \multicolumn{3}{|c|}{ Basic-adjusted model $(n=1027)$} & \multicolumn{3}{|c|}{$\begin{array}{l}\text { Fully-adjusted model }(\mathrm{n}=1021, \text { Model } \\
\left.\qquad \mathrm{R}^{2}=0.45\right)\end{array}$} \\
\hline & $\beta(95 \% \mathrm{CI})$ & $\mathrm{p}$ & $\begin{array}{l}\text { Partial } \\
\mathrm{R}^{2}(\%)\end{array}$ & $\beta(95 \% \mathrm{CI})$ & $\mathrm{p}$ & $\begin{array}{l}\text { Partial } \\
\mathrm{R}^{2}(\%)\end{array}$ & $\beta(95 \% \mathrm{CI})$ & $\mathrm{p}$ & $\begin{array}{l}\text { Partial } \\
\mathrm{R}^{2}(\%)\end{array}$ \\
\hline RP peak (mmHg) & $1.54(0.85$ to 2.23$)$ & $<0.001$ & 1.2 & $1.05(0.32$ to 1.78$)$ & 0.005 & 0.8 & $-0.47(-1.15$ to 0.20$)$ & 0.2 & 0.2 \\
\hline MAP (mmHg) & & & & & & & $5.80(5.14$ to 6.46$)$ & $<0.001$ & 22.8 \\
\hline $\begin{array}{c}\text { RP integral }(\mathrm{mmHg} / \mathrm{s}) \\
\text { MAP }(\mathrm{mmHg})\end{array}$ & 7.57 (4.95 to 10.19$)$ & $<0.001$ & 2.1 & $5.79(2.89$ to 8.69$)$ & $<0.001$ & 1.5 & $\begin{array}{l}-2.04(-4.81 \text { to } 0.72) \\
5.85(5.17 \text { to } 6.53)\end{array}$ & $\begin{array}{l}0.2 \\
<0.001\end{array}$ & $\begin{array}{l}0.2 \\
22.1\end{array}$ \\
\hline XSP peak (mmHg) & 2.48 (1.85 to 3.10$)$ & $<0.001$ & 3.8 & $0.47(-0.25$ to 1.19$)$ & 0.2 & 0.2 & $0.04(-0.59$ to 0.67$)$ & 0.9 & 0.01 \\
\hline MAP (mmHg) & & & & & & & $5.67(5.03$ to 6.30$)$ & $<0.001$ & 23.3 \\
\hline XSP integral (mmHg/s) & $10.8(7.51$ to 14.04$)$ & $<0.001$ & 2.6 & $2.37(-1.36$ to 6.09$)$ & 0.2 & 0.2 & $0.95(-2.31$ to 4.20$)$ & 0.6 & 0.03 \\
\hline MAP $(\mathrm{mmHg})$ & & & & & & & $5.66(5.03$ to 6.30$)$ & $<0.001$ & 23.3 \\
\hline
\end{tabular}

$\beta$ refers to unstandardised beta coefficient as the $\mathrm{cm} / \mathrm{s}$ difference in carotid-femoral pulse wave velocity per unit increase in reservoir pressure parameters and blood pressure. CI, confidence interval. $\mathrm{p}$ value is for the unstandardised $\beta$. Model $\mathrm{R}^{2}$ is the unadjusted model $\mathrm{R}^{2}$ as a proportion. Partial $\mathrm{R}^{2}(\%)$ is the proportion of total variance in carotid-femoral pulse wave velocity explained by individual risk factor. Basic-adjusted models adjust for age, sex, BMI, heart rate, smoking, diabetes and high-density lipoprotein cholesterol.

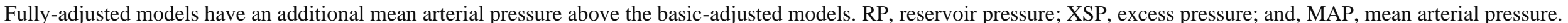

